\title{
Pierre Bourdieu: Un auto-análisis no biográfico
}

\author{
Franck Poupeau* \\ Hugo José Suárez**
}

\begin{abstract}
Resumen
En el artículo se recorre la trayectoria social de Pierre Bourdieu, intentando cruzar posición, contexto y obra. Se aborda su estancia en Argelia, su regreso a Francia y el campo intelectual de los años 60; sus iniciativas académicas e implicanciones políticas en las décadas posteriores $\mathrm{y}$, finalmente, su visión del mundo a finales de siglo, con las respectivas tomas de posición e intervenciones del sociólogo. El documento toma como base el Esbozo de un autoanálisis, que fue el libro póstumo de Bourdieu, e intenta, como lo sugiere el propio autor, no construir una biografía sino, más bien, situar una trayectoria en distintos momentos del campo académico y político que le tocó vivir.
\end{abstract}

Palabras clave: Pierre Bourdieu, sociología y política, autoanálisis sociológico.

\begin{abstract}
The article covers the social trajectory of Pierre Bourdieu, trying to cross position, context and work. Addresses his stay in Algeria, he returned to France in the field of intellectual 60s; implicanciones its academic initiatives and policies in the subsequent decades, and finally, his vision of the world at the end of the century, with the respective positions adopted and public sociologist. The document is based on the outline of the self, which was the posthumous book of Bourdieu, and tries, as suggested by the author himself, not to build a biography, but rather putting a track record at various times throughout the academic and political he lived.
\end{abstract}

Key words: Pierre Bourdieu, sociology and political, sociological self.

Doctor en Sociología por la Escuela de Altos Estudios en Ciencias Sociales de París. Miembro del Centro de Sociología Europea (Francia). Actualmente investigador del Instituto Francés de Estudios Andinos (IFEA, La Paz, Bolivia). Director editorial de la revista Actes de la recherche en sciences sociales, creada por Bourdieu en 1975. Correo electrónico: franck.poupeau@gmail.com

* Doctor en Sociología por la Universidad Católica de Lovaina. Investigador del Instituto de Investigaciones Sociales de la UNAM. Miembro del Sistema Nacional de Investigadores (México) nivel I. Correo electrónico: hugojosesuarez@yahoo.com y hugojose@unam.mx 


\section{Introducción}

Hasta los últimos días de su vida, Bourdieu fue un provocador. Su propia trayectoria fue un acto de rebeldía contra el discriminador sistema educativo francés, y en su obra no dejó de desarmar las razones del poder en distintos campos: las formalidades irrisibles del homo académicus, la irracionalidad de la razón neoliberal, la arrogancia de la distinción elitista, etc. Como exigente sociólogo fiel a la disciplina, se empeñó en observar no importa que ámbito de la vida social desde los lentes del oficio. Construyó científicamente cualquier problema, desde la opción de los gustos, hasta la puesta en práctica de las estructuras simbólicas. Una vida, cualquiera que sea, tenía que ser tratada de la misma manera: es lo que emprende en Esquisse pour une auto-analyse (Bourdieu, 2004), texto escrito en el segundo semestre del 2001 -meses antes de su muerte- que guiará el presente artículo.

Bourdieu fue reticente a escribir biografías o a que le hicieran una. Cuenta que, cuando algún estudioso le propuso narrar su vida, le sugirió que buscara un tema más interesante e importante. De hecho, en el inicio del texto señalado, el autor precede la frase "esto no es una autobiografía", y continúa contando pedazos de su recorrido. Años antes Bourdieu criticó la "historia de vida" como una noción "del sentido común que se ha introducido de contrabando en el mundo científico" (Bourdieu, 1997: 74); este concepto suele ser utilizado como si una existencia fuera una historia cronológica, una sucesión lineal de acontecimientos en etapas que confluyen a un punto de llegada. El enfoque sobreentiende la continuidad secuencial y relativamente coherente de la vida: toda persona nace, crece, actúa y muere siguiendo un mismo file rouge que, desde un momento dado, lo acompaña hasta el fin de sus días; así el concepto se acerca a un curriculum vitae con contenidos mejor desarrollados. El autor propone otra entrada. Una "historia de vida" no puede ser un fin en sí mismo sino que

“lleva a elaborar la noción de trayectoria como serie de las posiciones sucesivamente ocupadas por un mismo 
agente (o un mismo grupo) en un espacio en sí mismo en movimiento y sometido a incesantes transformaciones. Tratar de comprender una vida como una serie única y suficiente en sí de acontecimientos sucesivos sin más vínculo que la asociación a un 'sujeto' cuya constancia no es sin duda más que la de un nombre propio, es más o menos igual de absurdo que tratar de dar razón de un trayecto en el metro sin tener en cuenta la estructura de la red, es decir la matriz de las relaciones objetivas entre diferentes estaciones. (...). Sólo cabe comprender una trayectoria (es decir el envejecimiento social que, aunque inevitablemente lo acompaña, es independiente del envejecimiento biológico) a condición de haber elaborado previamente los estados sucesivos del campo en el que ésta se ha desarrollado, por tanto el conjunto de relaciones objetivas que han unido al agente considerado (...) al conjunto de los demás agentes comprometidos en el mismo campo y, enfrentados al mismo espacio de posibilidades..." (Bourdieu, 1997: 82)1.

La complejidad de tomar una existencia seriamente desde la sociología, implica hacer entrar en juego los múltiples elementos que la componen tanto en lo individual como en su correlación con el lugar social donde se inserta. Este enfoque se opone a considerar una vida como una bitácora de metáforas personales. Esa tarea es la que Bourdieu emprende en Esquisse..., es decir, en lugar de comenzar contando su infancia en Béarn (que lo hace al final del texto), se dedica a construir los distintos campos por los cuales transitó en varios momentos: retoma su historia "como si fuera cualquier otro objeto" (Bourdieu, 2004: 12). Cierto, "com-

Complementa el autor: "Los acontecimientos biográficos se definen como inversiones a plazo y desplazamientos en el espacio social, es decir, con mayor precisión, en los diferentes estados sucesivos de la estructura de la distribución de las diferentes especies de capital que están en juego en el campo considerado. El sentido de los movimientos que llevan de una posición a otra (...) se define, a todas luces, en relación objetiva mediante el sentido en el momento considerado de estas posiciones dentro de un espacio orientado" (Bourdieu, 1997: 82). 
prender, dice Bourdieu, es comprender el campo con el cual o contra el cual estamos hechos" (2004: 15):

El efecto del campo se ejerce por un lado a través de la confrontación con las tomas de posición de todo o de parte de aquellas que están también comprometidas en el campo (y que son encarnaciones distintas, y antagónicas de la relación entre un habitus y un campo): El espacio de los posibles se realiza en los individuos ejerciendo una 'atracción' o una 'repulsión' que depende de su 'peso' en el campo, es decir de su visibilidad, y también de la mayor o menor afinidad de los habitus que los llevan a encontrar 'simpáticos' o 'antipáticos' su pensamiento y su acción" (Bourdieu, 2004: 36)

En lo que sigue, nos concentraremos en cuatro posiciones de la trayectoria de Pierre Bourdieu: su intenso paso por Argelia, su consagración académica, su visión de la sociología del poder y de la educación, y finalmente su militancia social de la década del 1990.

\section{Argelia en la sociología del sociólogo}

Luego de la obtención de la "agregación" en filosofía en 1954 -grado que lo habilita a dar clases en el sistema francés y de particular prestigio en la vida académica- y de pasar por la Facultad de Letras de París de la Escuela Normal Superior (1951-1954), Bourdieu parte a Argelia a realizar su servicio militar. Se queda en ese país de 1955 a1960. El período que le toca vivir es de particular agitación por la Guerra de Argelia. El debate sobre el futuro argelino resuena en París y se convierte en uno de los temas de la agenda intelectual; es en este contexto que hay que comprender cómo el trabajo de Bourdieu en ese país es una forma de intervención política específica.

Argelia, antes de la independencia, representa tres departamentos franceses donde viven más de un millón de europeos y 
cuya administración está confiada al Ministerio del Interior. Los nueve millones de "ciudadanos argelinos", cuyos ingresos son, en promedio, veinte veces inferiores a los de los europeos, participan en un colegio separado, y sólo el 15\% de los niños musulmanes están escolarizados. La Guerra de Independencia, que comienza en noviembre de 1954, polariza durante varios años la vida política e intelectual francesa, provocando la caída de seis presidentes del Consejo y el desmoronamiento de la IVa República. El Frente Republicano, que ha llevado en 1956 a Guy Mollet y a los socialistas al poder, conduce a una política que acentúa la represión, especialmente con la ley sobre los poderes especiales de marzo de 1956. Esta política no deja de suscitar múltiples reacciones entre los intelectuales; como sugiere Pierre Vidal-Naquet (1998), a pesar de la diversidad de las formas de compromiso, la denuncia de la represión y la tortura constituyeron las causas más ampliamente defendidas por los diversos comités de sostenimiento a los argelinos. Periódicos como France Observateur, L'Express, Témoignage Chrètien o Le Monde emprenden en la época una batalla por la información. En la punta de ese combate, las ediciones de Minuit, dirigidas por Jérôme Lindon, publican La Question, de Henri Alleg (1958), y Déserteur, de Maurienne (1960), lo que desencadenará múltiples embargos por incitación a la desobediencia y atentado a la seguridad del Estado. Entre las figuras sobresalientes de la escena intelectual, Albert Camus, dividido entre el rechazo de las posiciones de los "ultras" de la Argelia francesa y su reticencia a admitir la independencia argelina, opta por callarse, mientras que Jean-Paul Sartre toma posición desde 1956 a favor de la lucha contra la "tiranía colonial". Preconiza la independencia argelina inmediata y la lucha junto al pueblo argelino, denunciando la tortura, testificando en los procesos, participando en las manifestaciones, firmando el "Manifiesto de los $121^{\prime 2}$, brindando su apoyo a la red Jeanson de ayuda al Frente de Liberación Nacional (FLN). La revista Les Temps Moder-

\footnotetext{
El Manifiesto de los 121 sobre el derecho a la insumisión en la guerra de Argelia, firmada por tantos intelectuales no llamaba a la insumisión o a la deserción sino que las "respetaba" y las juzgaba "justificadas"; proclamaba solemnemente que la causa del pueblo argelino era la de todos los hombres libres (Vidal-Naquet : 1998).
} 
nes, de la cual Sartre es el director, deviene el órgano del tercermundismo laico, y el libro de Frantz Fanon, Les Damnés de la terre (1961), que él prologa, le dan la ocasión de afirmar su anticolonialismo y de justificar una violencia, que supuestamente debe constituir, para el colonizado, el "medio para recomponer su naturaleza humana". El activismo sartreano quiere contrarrestar la tibieza de los partidos y sindicatos de izquierda. En el campo de la derecha liberal, Raymond Aron condena toda acción ilegal y clandestina, pero su obra Tragédie algérienne (1957) es favorable a la independencia, por lo que es desacreditado en el periódico donde escribe, Le Figaro, dirigido por Pierre Brisson, favorable a la Argelia francesa.

Cuando llega a Argelia en 1955 para hacer su servicio militar, Bourdieu ocupa enseguida un puesto de asistente de filosofía en la Facultad de Letras y no lo deja hasta abril de 1960, cuando Raymond Aron le propone enseñar en la Sorbona. Durante esos años en Argelia, Pierre Bourdieu emprende investigaciones etnológicas en Kabilia en condiciones descriptas por su estudiante y colaborador, Abdelmalek Sayad, como precarias y difíciles. Lo que Bourdieu llamará luego "el choque de Argelia" lo incita a escribir su primer libro, Sociologie de l'Algerie (1958), "en una lógica militante" -la edición norteamericana, de Beacon Press, presenta en la cobertura la bandera argelina, incluso antes de que la independencia fuera proclamada- iluminada por un conocimiento de la realidad argelina que no disponían muchos de los intelectuales parisinos. Su intención era "contar a los franceses, sobre todo de izquierda, lo que realmente sucedía en un país del cual ignoraban casi todo" (Bourdieu, 2004: 57).

Las dos primeras intervenciones políticas de Pierre Bourdieu son recopiladas en 1961 por Esprit y en 1962 por Les Temps Modernes, dos de las revistas más influyentes de la época, con las cuales no comparte forzosamente sus orientaciones ${ }^{3}$. Construidos en un

Paralelamente Pierre Bourdieu publica otros artículos en revistas más académicas como "Guerre et mutation sociales en Algérie", Études méditerranéennes, primavera de 1960, no 7, pp. 25-37; y “La hantise du chômage chez l'ouvrier algérien. Prolétariat et système colonial", Sociologie du travail, diciembre de 1962, nº 1, pp. 313-331. 
segundo plano etnográfico y siendo el resultado de varios meses de investigación en terreno, esos textos buscan romper con un uso apocalíptico de la etnología para hacer con ellos un instrumento de lucha simbólica. Analizan los efectos desestructurantes de la situación colonial, rechazando la neutralidad axiológica como pretexto a la falta de compromiso ${ }^{4}$.

En este contexto el autor se pregunta: ¿cómo se vive la transición de una sociedad esencialmente rural- hacia una racionalidad económica de mercado? ¿Qué implicaciones analíticas tiene el proceso? Bourdieu observa que "un sistema económico supone la existencia de un sistema determinado de actitudes con respecto al mundo y con respecto al tiempo" (1963: 24-25); existe una correspondencia entre las estructuras económicas y las estructuras simbólicas (sea capitalista o precapitalista). El proyecto de una sociedad capitalista requiere fundamentalmente "una estructura de conciencia temporal y un ethos correlativo (...) capaz de asegurar su éxito" (Bourdieu, 1963: 26). En Argelia precisamente se confrontan, en el análisis del autor, dos racionalidades distintas que se diferencian en los siguientes aspectos:

- La percepción del tiempo. La sociedad tradicional administra una noción temporal complementaria a la visión de la eternidad y del presente inmediato ("has como si deberías vivir eternamente, has como si deberías morir en un instante" (Bourdieu, 1963: 27), para lo cual se vincula la acción preponderantemente con la tradición, los imperativos y obligaciones sociales y la pertenencia a una comunidad. La sociedad moderna considera al presente como un momento de previsión donde se debe anticipar lo que podrá venir en el futuro; se requiere así construir un proyecto individual -privado de una lógica colectiva- tomando en cuenta los posibles imaginados realizando una serie de abstracciones para prever inclemencias no controlables.

4 Ver Travail et travailleurs en Algérie (con A. Darbel, J.-P. Rivet y C. Seibel), Mouton, París-la Haya, 1963; y Le Déracinement (con A. Sayad), Minuit, Paris, 1964. 
- La moneda y el trueque. La moneda se caracteriza por su indeterminación: "[es un] instrumento que sirve a quien sea, en cualquier lugar, para cualquier operación de intercambio"; tiene en sí misma una "infinidad de usos posibles" y de momentos para hacerlo, lo que conduce a un grado de abstracción. Por el contrario "las mercancías se intercambian en el trueque sobre la base de equivalencias establecidas por la tradición, tienen en ellas mismas su uso potencial, revelan su propio valor fundado sobre su cualidad de bien de uso, susceptibles de ser inmediatamente utilizadas, y no dependen, a diferencia de la moneda, de condiciones exteriores" (Bourdieu, 1963: 32)

- El crédito y el intercambio de dones. Una de las instituciones más difíciles de introducir por la colonización capitalista es la noción de crédito, pues "actúa en función de un futuro abstracto, definido por un contrato escrito que garantiza todo un sistema de sanciones y normas racionales". El intercambio de dones funciona más bien con base en la moral, el honor, el sentimiento de comunidad que hace que se genere confianza en los "hombres de honor fieles a sus compromisos", sin requerir de documentos firmados (Bourdieu, 1963: 35-36). Mientras que el crédito requiere del futuro, instituciones crediticias y un trato impersonal de las relaciones; el don reposa en el principio de solidaridad fraternal, intercambio de servicios, buena fe ("es un hombre de palabra"), integridad y vinculación operativa con el presente.

- El sentido del trabajo. Para el capitalismo el trabajo está orientado a la acumulación de capital luchando y dominando la naturaleza. En la sociedad tradicional el trabajo tiene el objetivo de satisfacer las necesidades primarias; la producción de bienes "permite al grupo subsistir y reproducirse biológicamente, y así revivificar los lazos, los valores y las creencias que crean la cohesión del grupo" (Bourdieu, 1963: 40) 
Así, la sociedad tradicional organiza sus ritmos temporales y espaciales a través de un calendario ritual sin necesidad de prevención racional. Es éste el que "garantiza la armonización de las conductas individuales" y el intercambio recíproco con los demás, lo que se reanima con el calendario del trabajo: los ritos "fundan la cohesión del grupo prohibiendo toda infracción a las expectativas colectivas al mismo tiempo que reduce al mínimo lo imprevisto" (Bourdieu, 1963: 42-43). El concepto teórico de correspondencia y complicidad -o grados de desfase- "entre las estructuras sociales y las estructuras mentales, entre las divisiones objetivas del mundo social (...) y los principios de visión y de división que los agentes le aplican" (Bourdieu, 1989: 7), desarrollado inicialmente en aquellos años gracias a sus observaciones etnográficas, le servirá para sus múltiples trabajos posteriores, aplicándolo al campo educativo, literario, religioso, etc.

Sin dar más razón ni al radicalismo verbal ni a las condenas humanistas y principistas que entonces hicieron de la revolución argelina un objeto de debates abstractos, la postura científica adoptada por Pierre Bourdieu le conduce a analizar las condiciones de acceso a la conciencia revolucionaria. El momento de la guerra es el de la revelación de la relación de violencia ejercida por el sistema colonial: más que oponer "enemigos", expone la revuelta de la sociedad dominada contra esta estructura de dominación. Ni guerra civil, ni guerra entre naciones, tampoco se agota en la lucha de una clase contra otra clase, porque toma por blanco el sistema de castas en cuanto tal -con armas que, por primera vez, no son solamente simbólicas-. Según Pierre Bourdieu, esta "revolución" revoluciona, a su vez, a la sociedad que la produce, en la medida en que hace perder a las conductas tradicionales el carácter de naturalidad que tenían, e impone a todos un desarraigo que se asemeja a la experiencia del inmigrado.

Esa experiencia-político intelectual lo marca en distintas direcciones para su reflexión posterior. De hecho es en Argelia donde gesta el primer habitus científico del oficio del quehacer 
sociológico. Las bases de su teoría del mundo social, que serán desarrolladas y afinadas en los siguientes cuarenta años de trabajo, están en las reflexiones argelinas; por eso él mismo decía que esas investigaciones más antiguas eran las que tenían, a la vez mayor actualidad (Bourdieu, 2003: 14); además, Argelia le permite "aceptarse a sí mismo" (Bourdieu, 2003: 42)5. No es casual entonces la cantidad de páginas que le dedica a ese pasaje de su vida en Esquisse... De múltiples maneras, en Argelia el profesor de filosofía se enfrenta a un trabajo etnográfico lo que le abre las puertas a construir otra manera de hacer sociología:

“Retornando de Argelia con una experiencia de etnólogo que, vivida en condiciones difíciles de una guerra de liberación, había marcado para mí una ruptura decisiva con la experiencia escolar, era portador de una visión bastante crítica de la sociología y de lo sociólogos, aquella del filósofo que se refuerza de aquella del etnólogo, y sobre todo, tal vez, una representación bastante desencantada o realista, de las posiciones intelectuales o colectivas de los intelectuales, para quienes la cuestión argelina se había constituido, a mi manera de ver, una excepcional piedra de choque" (Bourdieu, 2004: 53-54).

Es ahí, en el contacto con esa realidad, que Bourdieu construye la "libido scienti un poco exaltada que me animaba y que se enraizaba en una suerte de pasión por todo lo que tocaba en ese país, su gente, sus paisajes, y también la sorda y constante sensación de culpabilidad y de revuelta delante de tanto sufrimiento e injusticia que no daba descanso, ni tenía límites" (Bourdieu, 2004: 64-65).

Al menos son tres las rupturas -y "conversiones"- del investigador en Argelia: rompe con el "imperialismo de la filosofía"

\footnotetext{
"La mirada de etnólogo comprehensivo que tuve sobre Argelia, pude tomarla sobre mí mismo, sobre la gente de mi propio país, sobre mis padres, sobre el acento de mi padre, de mi madre, y recuperar todo esto sin drama, que es uno de los grandes problemas de todos los intelectuales desarraigados, encerrados entre la alternativa del populismo o por el contrario de la vergüenza del sí ligada al racismo de clase" (Bourdieu, 2003: 42).
} 
y, enfrentado con la realidad cotidiana, construye las primeras herramientas sociológicas para la interpretación de los espacios sociales; se ve obligado a pensar la situación de hacer sociología en situaciones límite -de agitación y riesgo- lo que lo conduce al concepto de reflexividad; y asume que parte de la misión del investigador social es construir científicamente problemas develando así las formas ocultas de dominación. Sobre ello sugiere Bourdieu:

“Llevar a cabo una investigación sociológica en situación de guerra obliga a pensar en todo, controlar todo, y particularmente lo que parece natural en la relación ordinaria entre investigador e investigado: la identidad de los investigadores, la propia composición de los investigadores -solo o de dos, cuando hay dos, un hombre y una mujer, un argelino y una francesa, etc. (...)-; más que nunca, se pone en duda el sentido mismo de la investigación por parte de los propios investigadores (¿no somos policías o espías?)" (Bourdieu, 2004: 68)

La obsesiva y terca necesidad de observar al científico que observa, de sospechar de él y por tanto de uno mismo, que posteriormente atravesará toda la obra de Bourdieu -lo que alguna vez llamará observación participante o reflexividad entendida como "objetivación científica del sujeto de la objetivación" (Bourdieu, 2004: 84$)^{7}$-, surge precisamente en el contexto de guerra que le toca investigar:

\footnotetext{
Años más tarde reflexionará: "trabajar, en el contexto de una Argelia en lucha por su independencia, en un análisis científico de la sociedad argelina, suponía tratar de comprender y de hacer comprender los fundamentos y los objetivos reales de esta lucha, objetivos que, estaba claro, eran socialmente diferenciados, antagónicos incluso, más allá de la unidad estratégica necesaria, e intentar así no, evidentemente, orientar su curso sino hacer previsibles, más difíciles por tanto, las probables desviaciones" (Bourdieu, 1991: 14)

7 En su clásico El sentido práctico, Bourdieu empieza diciendo: "El progreso del conocimiento supone, en el caso de la ciencia social, un progreso en el conocimiento de las condiciones del conocimiento; exige de este modo retornos obstinados sobre los mismos objetos (...), que son otras tantas ocasiones para objetivar más completamente la relación objetiva y subjetiva con el objeto" (1991: 13).
} 
"Sólo se consigue sobrevivir, en el sentido estricto, en tal situación (...) con el precio de una reflexividad permanente y práctica que es indispensable, en las condiciones de urgencia y de riesgos extremos, para interpretar y apreciar instantáneamente la situación y movilizar, más o menos concientemente, los saberes y el saber-hacer adquiridos en la primera experiencia social" (Bourdieu, 2004: 69)

Luego de esos años intensos en investigación y formación, Bourdieu nunca deja el trabajo de campo, "haciendo observaciones más o menos sistemáticas" (Bourdieu, 2004: 64) a lo largo de toda su vida.

\section{Crítica de las formas de distinción y campo intelectual en los años 1960}

De regreso en Francia, después de haber sido "maître de conférences" de sociología en la Facultad de Letras de Lille (19611964), Pierre Bourdieu se instala en París como director de investigaciones en la Escuela Práctica de Altos Estudios. Asume el rol de secretario general del Centro de Sociología Europea (CSE), lanzado por Raymond Aron en 1960, gracias a una subvención de la Fundación Ford. A lo largo de esos años, se constituye un grupo de investigadores, entre los cuales están Luc Boltanski, Robert Castel, Jean-Claude Chamboredon, Patrick Champagne, Yvette Desault, Claude Grignon, Rémi Lenoir, Francine Muel-Dreyfus, Jean-Claude Passeron, Louis Pinto, Monique de Saint-Martin y Dominique Schnapper. Sus estudios y publicaciones están dedicadas al sistema de enseñanza, a los intelectuales, a las prácticas culturales ligadas a los museos y a la fotografía ${ }^{8}$. En el año 1964 crea la colección "Le sens comun" en las ediciones de Minuit, como respuesta a la necesidad de consolidar una estructura de publicación relativamente autónoma cuya política editorial fue$8 \quad$ Ver Un art moyen (1965); L'amour de l'art (1966); Le Partage des bénéfices (1966) ; Le
Métier de sociologue (1968). 
ra, a la vez, científicamente ambiciosa (las traducciones de obras maestras de la tradición crítica francesa y extranjera vendrán a reforzar la serie de los grandes estudios que daban a luz los miembros de ese colectivo sociológico) y preocupada por escapar del confinamiento erudito para abrirse a un público lector más vasto y marcado por ciertas expectativas políticas asociadas a la "etiqueta Minuit". En esta colección Pierre Bourdieu publica con Jean-Claude Passeron, en el mismo año, Les Héritiers, libro en el cual Raymond Aron verá más tarde uno de los catalizadores de Mayo del 68. Con Aron, Bourdieu tuvo una relación muy cercana y de respeto e intercambio intelectual. Alguna vez Aron le dijo: "desde muy temprano tienes un sistema de conceptos" (Bourdieu, 2004: 48), y pasaban noches enteras discutiendo textos e ideas. Pero distintas circunstancias -particularmente su posición frente a los eventos del 68- condujeron a un distanciamiento.

El ambiente intelectual francés se define, preponderantemente, por Jean Paul Sartre. Bourdieu critica no propiamente el contenido de la obra sartiana ni su militancia en causas sociales, sino el hecho de haber construido alrededor suyo la figura del "intelectual total" que contribuía a la "mitología del intelectual libre que tiene garantizado el reconocimiento eterno de todos los intelectuales" (Bourdieu, 2004: 37). De alguna manera la toma de posición política de Sartre iba de la mano de la consolidación de la figura del académico iluminado y del modelo de "filósofo normalista francés de los años 50" (Bourdieu, 2004: 37). Precisamente una de las batallas de Bourdieu fue la denuncia de las nuevas formas de nobleza de Estado que se generaban a partir de la institución universitaria, y el culto al filósofo y a la filosofía como disciplina más valorada que las demás, lo que no hacía más que reproducir los arcaicos modos de distinción y jerarquía en la sociedad francesa. Sartre construye una criatura mítica de la misión del intelectual y del compromiso social. Así las cosas, el filósofo es el mejor ejemplo de la legitimidad y culto al académico virtuoso. Entre otras razones, la opción de Bourdieu por la sociología y 
no por la filosofía es una manera de criticar esa forma estatal de distribución de la consagración.

Sin embargo, en la dupla Sartre-Aron -clásica del debate intelectual francés de los 70-, Bourdieu no opta por el segundo a pesar de su cercanía, sino que busca los puntos de encuentro entre ambos en lugar de las diferencias: son producto de la élite escolar triunfante que poseen un reconocimiento innegable, provienen de "un concurso escolar de reclutamiento (la agregación en filosofía) que es una instancia de consagración intelectual" (Bourdieu, 2004: 39), creen en la institución universitaria y en los "poderes de la inteligencia" como transformación de la humanidad. Las afinidades intelectuales de Bourdieu no son las figuras espectaculares de la academia sino aquellos que contribuían al "análisis riguroso de la génesis de los conceptos científicos y los obstáculos históricos para su emergencia" (Bourdieu, 2004: 41), como Georges Canguilhem que, en su libro Études d'histoire et de philosophie des sciences (1968) se preocupaba de la seriedad en la investigación científica, lo que no implicaba un aislamiento de lo social en reflexiones epistemológicas, sino que era esforzarse por afinar las herramientas para mirar la sociedad y no jugar y disfrutar de la legitimidad del "ser filósofo". En lugar de gozar de los beneficios de estar en la cima del campo, Bourdieu sugiere analizarlo: "existen muchos intelectuales que interrogan el mundo; hay pocos intelectuales que interrogan el mundo intelectual" (Bourdieu, 2004: 37).

\section{Sociología crítica y nuevas iniciativas intelectuales en los años 1970 - 80}

En enero de 1971 en Arrás, Pierre Bourdieu da una conferencia titulada "La opinión pública no existe", que será retomada en Les Temps Modernes dos años más tarde (Bourdieu, 1973). Ese texto es fundacional en varios aspectos: la crítica que efectúa a los sondeos de opinión y a su uso, se dirige, a la vez, a los investigadores que los realizan y a los políticos que hacen de ellos un argumento 
de autoridad. La consideración de las no respuestas en los sondeos plantea el problema de las competencias necesarias para hablar de política y de la desposesión sufrida por aquellos que se remiten a mandatarios para representar su palabra política. Esta crítica sorprende, en un primer momento, a los politólogos que aceptan primero el principio sobre una base metodológica, antes de reaccionar sobre bases políticas:

“En el proceso hecho a los sondeos en nombre de la democracia, me sitúo resueltamente del lado de la defensa. Ello se relaciona en mucho, sin ninguna duda, con mi concepción de la democracia, que es incurablemente liberal. [...] Es una concepción que descansa en la fe en el sufragio universal. [...] Las principales críticas formuladas a los sondeos de opinión podrían igualmente ser utilizadas contra el sufragio universal. [...] En los dos casos, se desconfía de las 'mayorías silenciosas' en nombre de las minorías que son las únicas que saben 'lo que quiere decir hablar'" (Lancelot: 1982, s/p)

Además, la crítica a los usos politológicos de "la opinión pública" constituye, según Pierre Bourdieu, una defensa de la autonomía de la sociología en el momento mismo en que los investigadores se encuentran subordinados a las demandas políticas y administrativas, cada vez más dominados por un polo de investigación aplicada cuyo principal representante, en la década de 1970, es Jean Stroetzel, entonces en posición dominante: profesor en París (donde enseña psicología social); dirige el Centro de Estudios Sociológicos y el Instituto Francés de Opinión Pública (IFOP), que ha desarrollado la técnica de los sondeos, importada de Estados Unidos; controla el acceso al Centro Nacional de Investigación Científica (CNRS) así como La Revue française de sociologie, una de las cuatro grandes revistas que dinamizan entonces a las ciencias sociales.

La legitimidad intelectual que dan los sondeos -"esta ciencia sin sabios"- a los mecanismos de dominación constituye, para Pie- 
rre Bourdieu, el fundamento de su crítica a los "doxósofos", esos profesionales de la fabricación de la opinión que producen una ideología conforme a los intereses de los dominantes. La crítica política debe pues acompañarse, según Bourdieu, con una sociología de los intelectuales utilizada como un arma simbólica contra las justificaciones seudo-científicas del orden social. Una empresa que genera resistencias, como lo testimonian la constancia de los argumentos expresados (especialmente sobre el tema del determinismo) en la polémica del sociólogo con ciertos intelectuales marxistas (La Nouvelle Critique) y con cristianos de izquierda (Esprit).

Por otro lado, mientras que en la década de 1970 se veía el ascenso de la izquierda francesa, una parte de los cuadros de la alta administración se pone al servicio de la "modernización" del capitalismo nacional. Esas transformaciones inducen un incremento de la empresa de los poderes políticos sobre el mundo intelectual: entre los intelectuales privados de poder temporal y los hombres de poder cuya autoridad se apoya cada vez más sobre competencias específicas se desarrollaba, desde la década de 1950, una población de "investigadores administrativos" y de "administradores científicos" pertenecientes a instituciones de investigación que responden a los encargos de la administración.

Frente a este panorama, Bourdieu funda, en 1975, la revista Actes de la recherche en sciences sociales que pretende contribuir a reforzar la autonomía de la sociología dotándola de un medio de difusión independiente, sometido sólo a las exigencias de los procedimientos de verificación y de crítica científica. Marcada por la voluntad de romper con el formalismo académico y la estandarización normalizante de la investigación, la política editorial Les Actes yuxtapone artículos "acabados", notas, memorias intermedias, documentos estadísticos, fotografías, facsímiles e historietas. Esta política científica en sociología no quiere solamente "deconstruir" los textos "sagrados" del mundo científico sino también "destruir los pretextos falsos y las evasivas forjadas por una visión religiosa del hombre de la cual las religiones reve- 
ladas no tienen el monopolio". Operando un "derrumbamiento de la jerarquía de los objetos consagrados" por una ciencia tan poco independiente de las demandas políticas como la sociología, donde la censura científica no es, con bastante frecuencia, sino una censura política oculta, la revista quiere cambiar completamente la oposición entre "el sacerdote de la gran ortodoxia académica" y "la herejía distinguida de los francotiradores de fogueo". En esta dirección, en el artículo inaugural de Les Actes, Bourdieu denuncia una jerarquía de las especialidades en el ámbito educativo que no sólo se podía observar en la distancia entre la filosofía y las demás disciplinas, sino en la propia elección de objetos de estudio de los investigadores sociales:

La jerarquía de los objetos legítimos, legitimables o indignos es una de las mediaciones a través de las cuales se impone la censura específica de un campo determinado que, en el caso de un campo cuya independencia con respecto a las demandas de la clase dominante está mal afirmada, puede ser la máscara de una censura propiamente política. La definición dominante de las cosas que vale la pena decir y de los temas dignos de interés es uno de los mecanismos ideológicos que hacen que cosas que valen la pena decir no sean dichas y que temas dignos de interés no interesen a nadie (Bourdieu, 1975: 4)

Así, los temas clásicos como el Estado, el poder, los movimientos sociales, son más valorados que la cultura popular, la etnografía, las prácticas rurales, etc. Se puede establecer una relación entre la construcción de objetos científicos y el orden político; entre la opción científica y la posición política. Bourdieu propone en la revista una variedad de temas hasta el momento poco considerados como dignos de estudio: la "alta costura", el automóvil, la historieta, la enseñanza técnica, el ejército, los trabajadores sociales, la retórica marxista, etc.

Paralelamente, en esos años Bourdieu desarrolla parte de sus obras más fundamentales que giran alrededor de las estructuras 
simbólicas, los sistemas de distinción y en general la teoría de los campos. Entre los textos más significativos tenemos La distinción (1979), y El sentido práctico (1980), que son, además de lo acumulado, los que tocan las puertas de su posición en El Colegio de Francia. En La distinción, Bourdieu demuestra que un objeto de estudio escurridizo como el gusto corresponde a una estructura social y cultural que lo determina y construye, y que, además, se relaciona con las distintas formas de posesionarse frente a otras esferas de la vida social como la política, la estética, la vida cotidiana, etc. Es decir que espacio social, principios de diferenciación y disposiciones simbólicas se encuentran articulados entre sí. Lo que analiza concretamente en esta investigación, inundada de datos empíricos, va de la mano de El sentido práctico, donde explica conceptos como habitus, estructuras, capital simbólico, campo, etc. ${ }^{9}$

El ingreso del investigador a la sociología de la educación tiene que ver con su propia posición que ocupó en las distintas esferas del sistema educativo francés. Recordemos que de adolescente, entre los 11 y 17 años, estudia internado en un liceo de Pau (al sur de Francia), donde fue sometido a las "regularidades monótonas" en un ambiente similar a las "instituciones totales" que describe Goffman en Los Asilos (1968) -según él mismo comenta-. Ahí vivió la experiencia del castigo preventivo, las represalias, la rebelión, la culpabilidad inculcada por las autoridades cuando alguien tenía un comportamiento inadecuado. La lógica de la sanción llevaba a puniciones colectivas donde todos pagaban por lo que uno había hecho, incentivando al control por parte de los demás ("vas a hacer que nos maten") y la autodenuncia ("denúnciate a ti mismo" -Bourdieu, 2004:122). Pero el mayor impacto escolar lo vivió como interno en el Liceo Louis-le-Grand de París (1948-1951) y en la Escuela Normal Superior (19511954). En ese lugar privilegiado quedaba más claro lo que vivió en Pau en términos de diferenciación entre el provinciano y los

\footnotetext{
Véase Wacquant, 2006: 6.
} 
hijos de funcionarios distinguidos. Mientras los parisinos mostraban su "elegancia burguesa" y las "pretensiones literarias de sus producciones, desde entonces concebidas como creación de escritores" (Bourdieu, 2004: 126), los originarios del mundo rural eran objeto de burla por el acento, su forma de vestir y comportamiento general. Estas formas de discriminación-legitimación adquirían mayor claridad en la Escuela Normal donde se aprendían las formalidades de la consagración en el mundo educativo francés: el aire aristocrático, la arrogancia, los "juegos del concurso", el "arribismo pequeño burgués"; características claves para convertirse en el futuro en "miembros eminentes de la jerarquía universitaria y las sucesivas encarnaciones del homo-academicus" (Bourdieu, 2004: 130).

Este antecedente explica su actitud cuando, en 1981, es elegido como profesor titular de la cátedra de sociología en el Colegio de Francia; su conferencia inaugural titula "Lección sobre la lección". En efecto, al asumir el lugar académico más prestigiado sufre una de las mayores contradicciones de su carrera: iba a ser coronado -con las formas solemnes de la nobleza intelectual- por las instancias que siempre había criticado. Por eso escogió desarrollar el tema que hablara del propio acto de consagración social en el cuál él era el protagonista principal: "tomar como objeto en mi lección el hecho de dar una lección inaugural, de consumar un rito de institución y así instaurar una distancia con el rol en el propio ejercicio del rol" (Bourdieu, 2004: 138). En su conferencia sitúa su pensamiento como un esfuerzo epistemológico por analizar las formas propias de la construcción del saber científico: propone hacer "sociología de la sociología". La sociología del sistema educativo se constituye en un instrumento para conocer y analizar "las categorías de pensamiento impensadas que delimitan lo pensable y predeterminan lo pensado", por eso "no hay crítica epistemológica sin crítica social" (Bourdieu, 2002: 10-11). 


\section{El sufrimiento social: Miseria del mundo y Razones para actuar (los años 90)}

A fines de la década de 1980, luego de su participación en el informe de expertos para el Estado, Pierre Bourdieu lanza un proyecto colectivo, La miseria del mundo, que aparece en 1993 y deviene rápidamente un trabajo de referencia en el seno de los movimientos sociales. Ese libro obtiene un enorme éxito público: se venden más de 80.000 ejemplares, es llevado al teatro y traducido a varias lenguas. El "Post-scriptum" del libro interpela directamente al cierre del mundo político sobre sí mismo y su olvido de la realidad social; y el título del libro parece responder al Primer Ministro Michel Rocard que, bajo el empuje electoral de un partido de extrema derecha -el Frente Nacional- respecto al "problema de la inmigración", había declarado en el periódico Le Monde del 24 de agosto de 1990: "Francia no puede acoger toda la miseria del mundo, pero debe saber tomar fielmente su parte".

Sin duda, se puede apreciar la ruptura que constituye la dirección de esta investigación colectiva, volviendo sobre el análisis que Pierre Bourdieu hiciera, dos años antes, de la solución política que el gobierno de Michel Rocard venía de aportar a las reivindicaciones independentistas de los Canacos ${ }^{10}$ : momento de retroceso en "una formidable crisis de la representación y delegación políticas". Según Pierre Bourdieu, es el principio mismo de la representación política lo que está en cuestión; o, más precisamente, "la usurpación legítima" de todo cargo público, "el misterio del ministerio", ese poder que el mandatario político toma de la delegación. Este análisis del ejercicio del poder no conduce, para Bourdieu, a la pasividad o a la resignación. Si ciertos gru-

10 Las luchas por la independencia de Nueva Caledonia habían conocido un episodio sangriento, entre las dos vueltas del escrutinio electoral presidencial, cuando el Primer Ministro y candidato Jacques Chirac ordenó la toma por asalto de la gruta de Ouvéa, donde los independentistas se habían parapetado con sus rehenes, lo que se tradujo en la masacre de los militantes del FLNKS. (Sobre la posición de Pierre Bourdieu, "Quand les Canaques prennent la parole. Entretien avec Alban Bensa", Actes de la recherche en sciences sociales, 1985, no 56, pp. 69-83). 
pos sociales han podido trabajar en la instauración del Estado de derecho, de la idea de servicio público o de interés general, es porque han encontrado allí beneficios de universalización ${ }^{11}$ :

“Una política eficaz y realista consistiría en ampliar ese principio de interés por lo universal a otros universos sociales, y en inventar estructuras institucionales para que los políticos tengan interés en la virtud. La moral política no puede caer del cielo: no está inscripta en la naturaleza humana. Sólo una Realpolitik de la razón y de la moral, puede contribuir a favorecer la instauración de universos donde todos los agentes sociales estarían sometidos -especialmente por la crítica- a una suerte de test de universalidad permanente [...]. La moral no tiene posibilidad de advenir, particularmente en política, más que si se trabaja en crear los medios institucionales de una política de la moral" (Bourdieu, 1994: 239).

El rol de la crítica pública se revela entonces determinante para forzar a los hombres políticos a ser lo que su función social les prescribe ser, es decir, a reducir "la distancia entre lo oficial y lo oficioso" y a "crear las condiciones de instauración del reino de la virtud civil". Ahora bien, esta crítica a las burocracias nacionales no conduce solamente a develar el sufrimiento social engendrado por las políticas neoliberales dirigidas por la propia izquierda. Está acompañada de una reflexión sobre las condiciones de la acción política de los intelectuales, cuya autonomía está amenazada por la influencia de una "tecnocracia de la comunicación" que refuerza el monopolio de los profesionales de la política sobre el debate público:

“El problema que planteo permanentemente es el de saber cómo hacer entrar en el debate público esta comunidad de científicos que tiene cosas que decir sobre la

Para una presentación más detallada, ver "Esprits d'État", in Raisons pratiques (1994: 99-146). 
cuestión árabe, sobre los barrios periféricos, el fular islámico... ¿Pues, quién habla [en los mass-media]? Son subfilósofos que tienen por toda competencia vagas lecturas de textos vagos, gente como Alain Finkielkraut. Llamo así a los pobres Blancos de la cultura. Son semi-sabios no muy cultivados, que se presentan como los defensores de una cultura que no tienen, para marcar la diferencia con los que aún tienen menos que ellos. Aquella gente se apropia del espacio público y expulsan de allí a los que tienen cosas que decir. Antes de hablar del "mal de los barrios periféricos", antes de proferir todas esas tonterías que se escuchan entre los intelectuales franceses jes necesario, en primer lugar, ir allá!. Los que así manifiestan veredictos hacen mal, porque dicen cosas irresponsables. Y, al mismo tiempo, desalientan la intervención de la gente que está en el terreno, que trabajan y que tienen cosas que decir. Actualmente, ellos constituyen uno de los grandes obstáculos para el conocimiento del mundo social"12.

Así, frente a un escenario de los noventa marcado por el sufrimiento social, el imperio de la razón tecnocrática, el monopolio de los medios, surge también la acción colectiva. En efecto, a finales de 1995 se desarrolla en Francia un movimiento social importante que recordó los agitados días del mayo del 68. Se dice que no se había vivido una movilización de ese tipo desde aquella paradigmática revuelta estudiantil. En este caso el actor había cambiado, se trataba de los "excluidos", un conglomerado humano compuesto por desempleados, migrantes sin papeles, obreros, mujeres, estudiantes, etc. Todo se desencadenó a partir del denominado "plan Juppé", nombre de la política gubernamental de reforma de la seguridad social (creación de un nuevo impuesto, limitación de gasto en salud, reforzamiento del rol del parlamen-

\footnotetext{
12 "Les intellectuels ont mal à l'Europe", entrevista con Michel Audédat, L'Hebdo, 14 de noviembre de 1991.
} 
to, etc.), que implicaba poner en riesgo una serie de conquistas sociales que venían de larga data.

Los distintos acontecimientos despertaron paralelamente al mundo intelectual que se encontraba dormido durante ya varias décadas. Se generó súbitamente un debate público tanto en la prensa como en las calles, donde los distintos investigadores y profesores universitarios empezaron a tomar partido. Se habla de un "regreso de los intelectuales" luego de "largos años de silencio". La situación obliga a los sociólogos, artistas o investigadores, a salir de sus propias discusiones y "tomar una posición sobre el fondo del debate en curso, sobre la política económica, sobre la situación económica y social"(Duval et. all., 1998: 11). Rápidamente se polariza el propio campo intelectual, entre aquellos que defendían el "plan Juppé" y quienes estaban en contra. En el primer grupo se encontraba la revista Esprit, la Fondation SaintSimon y algunos investigadores reconocidos. La respuesta no se hizo esperar, una serie de profesores, entre los cuales estaban Pierre Bourdieu, Samir Amín, Patrick Champagne, Regis Debray, Claude Dubar, Michael Lowy, publicó un comunicado titulado "llamada de los intelectuales en apoyo a los huelguistas":

Nos reconocemos plenamente en ese movimiento que no defiende intereses particulares sino que es una defensa de los logros más universales de la República. Luchando por sus derechos sociales, los huelguistas luchan por la igualdad de derechos entre todos: mujeres y hombres, jóvenes y viejos, desempleados y asalariados, trabajadores, asalariados de lo privado y de lo público, inmigrantes y franceses (...). Invocamos a todos los conciudadanos a asociarse a este movimiento y a la reflexión radical sobre el futuro de nuestra sociedad..." (Duval et. all., 1998: 19).

Es a partir de aquellas movilizaciones que se empieza a conformar un grupo más permanente y menos coyuntural que se 
denominará "Razones para actuar", y comenzarán una serie de actividades político-académicas en favor de los diferentes movimientos sociales. La participación de Bourdieu y su grupo en los medios empieza a cobrar una importancia sistemática, y suscita reacciones a favor y en contra. Se dedican números enteros de Le Nouvel Observateur, Le Magazine Littéraire, Esprit, artículos en la portada de Le Monde, Le Monde Diplomatique, Liberation y otros medios de difusión; se organizan conferencias y debates sobre el tema. Vuelve la pregunta que había estado guardada en el cajón de los recuerdos: ¿cuál es el rol del intelectual en la vida social?

Lo particularmente novedoso de este movimiento es que la respuesta sería distinta a aquella que dieran Sartre o Foucault en su momento. Ahora no se trata de una relación orgánica a un partido o institución, sino más bien una unidad social como reacción espontánea a la fragmentación liberal. De ahí nace una propuesta asociativa diferente que se llamará "razones para actuar" con la intención de proponer un "intelectual colectivo" capaz de reunir informaciones y análisis de la situación social general para resistir a la hegemonía del pensamiento neoliberal vinculado con los movimientos sociales. En 1998 Bourdieu presenta el libro Contrafuegos, cuyo matiz es muy político e intenta "servir a la resistencia contra la invasión neoliberal". El documento fue una recopilación de artículos, entrevistas o intervenciones en actos públicos donde el autor toma una posición política radical de deslegitimación del discurso neoliberal. En el texto tenemos a Bourdieu sociólogo-político en acción. Se enfrenta contra las teorías de la acción racional que pretenden tener el monopolio de la razón y que, a través de los tecnócratas del FMI o del Banco Mundial, delimitan y dirigen los mandamientos del neoliberalismo (Bourdieu, 1998: 25). Aquellos miembros de la "nobleza del Estado", que se creen autorizados para gobernar gracias a los nuevos bienes simbólicos que poseen (estudios en escuelas norteamericanas, vínculos con grandes transnacionales, etc.), no hacen otra cosa que someter a la sociedad a la lógica del mercado y del consumo, convirtiendo el bien público en bien privado. Esta "tiranía de los expertos" 
crea un nuevo Leviatán: "El mercado financiero, y no se dispone negociar -con movimientos sociales o intelectuales- sino 'explicar', hay que romper con la nueva fe en la inevitabilidad histórica que profesan los teóricos del liberalismo"(Bourdieu, 1998: 31).

Bourdieu acusa a la "ortodoxia tecnocrática" de poner en riesgo grandes logros que pertenecen a la humanidad: los derechos sociales que son bienes públicos y por tanto pertenecen a todos. Critica a los medios de comunicación y particularmente a los intelectuales mediáticos que se encargan de "hacer la opinión" imponiendo su propia perspectiva empleando "palabras que no dicen nada -como 'aldea planetaria', 'mundialización'- pero mediante las cuales se transmite toda una filosofía, una visión del mundo, palabras que engendran el fatalismo y la sumisión" (Bourdieu, 1998: 63). Opta por los movimientos sociales que luchan por una "civilización asociada a la existencia del servicio público, de la igualdad republicana de los derechos, derechos a la educación, la salud, la cultura, la investigación, el arte, y, sobre todo, al trabajo"(Bourdieu, 1998: 30). Finalmente, llama a la resistencia contra el neoliberalismo y la "revolución conservadora" y promueve la defensa del "interés público" a partir de distintas instancias colectivas: sindicatos, organizaciones de la sociedad civil, partidos políticos, grupos de investigación, colectivos de reflexión, etc. De 1995 hasta su muerte, Bourdieu no dejó de tener manifestaciones de apoyo a movimientos sociales: "La historia social nos enseña que no hay política social sin un movimiento social capaz de imponerlo (y no es el mercado como nos intenta hacer creer, sino el movimiento social el que ha "civilizado" la economía del mercado, contribuyendo en gran medida a su eficacia)" (Bourdieu, 1999: 16).

\section{Conclusión: el sociólogo herético}

Bourdieu se esforzó por escapar de las formas del poder (académico, político, social, temático) y buscó, apoyado en la rigurosidad científica y la reflexividad constante sobre el saber sociológico, 
adentrarse en territorios no consagrados. Siempre desde el margen, con una extraña "mezcla de timidez agresiva con brutalidad escandalosa, hasta furiosa" (Bourdieu, 2004: 115), ofreció investigaciones que, abordando distintos objetos concretos, quebraran con el sentido común. Este sociólogo incómodo y que incomoda, consideró a que el rol de la disciplina era "develar cosas ocultas (...) que ciertos individuos o ciertos grupos prefieren esconder o esconderse porque ellas perturban sus convicciones o sus intereses" (Bourdieu, 1997b: 65).

¿Por qué Bourdieu se esfuerza en ser un sociólogo herético? Probablemente porque, como él mismo explica, su propia trayectoria está marcada por un desfase entre "una elevada consagración escolar y una baja extracción social, o sea un habitus clivé movido por tensiones y contracciones" (Bourdieu, 2004: 127). Él es el ejemplo más paradójico del sistema educativo francés: siendo de familia de provincia y condenado por las estructuras sociales a ser un "don nadie" en el ámbito intelectual, llega a la posición de máximo prestigio de la academia. Era de esperarse, entonces, que en lugar de continuar con las formalidades de legitimidad intelectual, pusiera en duda el propio sistema que, contra toda tendencia "natural", lo consagró. Por eso sus objetos de estudio fueron lo que la sociedad consideraba como trivial. Pero para profundizar en esta reflexión, habría que convertir a Bourdieu y su obra en un objeto de investigación, cayendo, en lo que él mismo criticaba: ocuparse de los temas hoy legítimos de la sociología. Parece más sabio, y como un homenaje al propio sociólogo, apuntar sus herramientas analíticas en otras direcciones.

\section{Bibliografía}

Alleg, Henri (1958). La Question, Ed. Minuit, Paris.

Aron, Raymond (1957). Tragédie algérienne, Ed. Plon, París.

Bourdieu, Pierre (2004). Esquisse pour une auto-analyse, Ed. Raison d'Agir, Paris.

(2003). Images d'Algérie, Ed. Actes Sud, París. 
(2002). Lección sobre la lección, Ed. Anagrama, Barcelona. (2002b). Interventions. 1961-2001. Science sociale et action politique, Ed. Agone, Paris.

(1999). "Pour un mouvement social européen", Le Monde Diplomatique, Juin.

(1998). Contre-feux, Ed. Liber-Raison d'agir, Paris.

$\left(1997^{\mathrm{a}}\right)$. Razones prácticas. Sobre la teoría de la acción, Ed. Anagrama, Barcelona.

(1997b). Capital cultural, escuela y espacio social, Ed. S. XXI, México D.F.

(1994). Raisons pratiques. Sur la théorie de l'action, Ed. Seuil, Paris. [Razones prácticas, sobre la teoría de la acción, Barcelona, Anagrama, 1997]

(1991). El sentido práctico, Ed. Taurus, Madrid.

(1989). La Noblesse d'Etat, grandes écoles et esprit de corps, Ed. Minuit, Paris.

(1979). La distinction. Critique sociale du jugement, Ed. Minuit, Paris.

(1975). "Méthode scientifique \& hiérarchie sociale des objets", in Actes de la recherche en sciences sociales, 1975, N. 1.

(1973). "L'opinion publique n'existe pas", en Les Temps modernes, $\mathrm{n}^{\circ} 318$, janvier.

(1970). La reproduction. Eléments pour une théorie du système d'enseignement (con Jean-Claude Passeron), Editions de Minuit, Paris.

(1968). Le Métier de sociologue (con Jean-Claude Chamboredon y Jean-Claude Passeron), Minuit, Paris. [El oficio de sociólogo, Siglo XXI, Buenos Aires, 1975]

(1966). L'amour de l'art (con A. Darbel y Dominique Schnapper), Minuit, Paris. [El amor al arte, Paidos, Buenos Aires, 2004]

(1966b). Le Partage des bénéfices (en colaboración con estadísticos y economistas del INSEE), Minuit, Paris

(1965). Un art moyen (con Luc Bolstanski, Robert Castel y Jean-Claude Chamboredon), Minuit, Paris. [La fotografía. Un arte intermedio, Nueva Imagen, México, 1979] 
(1964). Le Déracinement (con A. Sayad), Minuit, Paris.

(1964b). Les héritiers. Les étudiants et la culture (Con JeanClaude Passeron), Ed. Minuit, París.

(1963). Travail et travailleurs en Algérie (con A. Darbel, J.-P. Rivet y C. Seibel), Mouton, París-la Haya.

(1963b). "La société traditionnelle, Attitude à l'égard du temps et conduite économique", in Sociologie du Travail, N. 1, p. 24-44.

(1962). "Les sous-prolétaires algériens", Les Temps Modernes, diciembre, \# 1999, pp. 1030-1051

(1962b). "La hantise du chomage chez l'ouvrier algérien. Prolétariat et système colonial", Sociologie du travail, diciembre, \# 1, p. 313-331

(1960). "Guerre et mutation sociales en Algérie", Études méditerranéennes, primavera, \# 7, pp. 25-37

(1957). Sociologie d'Algérie, PUF, París.

Canguilhem, Goerges (1968). Études d'histoire et de philosophie des sciences, Vrin, Paris.

Duval, J., Gaubert, C., Lebaron, F, Marchetti, D., Pavis, F. (1998). Le "décembre» des intellectuels français, Ed. Liber-Raison d'agir, Paris

Goffman, Erving (1968). Asiles, Ed. Minuit, París.

Fanon, Frantz (1961). Les Damnés de la terre, Ed. Maspero, Paris

Lancelot, Alain (1982). Communication au colloque "Sondages et droits du public", Université du Québec.

Maurienne (1960). Déserteur, Ed. Minuit, Paris.

Vidal-Naquet, Pierre (1998). Mémoires II, Seuil-La Découverte, Paris

Wacquant, Loïc (2002). "O legado sociológico de Pierre Bourdieu: duas dimensoes e uma nota pessoal", en Revista de Sociología e Política, N. 19. Disponible en página web: www.scielo.br/pdf/rsocp/ n19/14625.pdf 